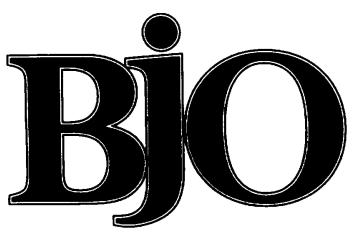

British Journal of Ophthalmology

Editorials

\title{
Towards the automatic interpretation of retinal images
}

Perhaps the most difficult tasks we can ask of computer systems is that of image analysis and interpretation. Diagnosticians working with images, whether they are ophthalmologists or radiologists, rapidly arrive at decisions based largely on experience. The history of computing with digital images has been successful where issues of quantitation are involved but we have shown only limited ability to define general purpose algorithms which, starting from scratch, produce clinically useful observations. Our systems may, for example, be able to determine the number, size, and distribution of microaneurysms at an acceptable level of sensitivity and specificity, but the mapping of this information unequivocally to a specific disease is much less amenable to naive computation. However, by directly relating disease with a set of symptomatic image features, artificial neural networks (ANNs) circumvent the need for logical reasoning between observation and diagnosis, introducing a range of powerful new techniques for clinical image interpretation.

The conventional approach to computing is based upon programmed instructions and stems from Babbage's analytical engine design of the 1840 s, Turing's proof, 100 years later, that such machines could perform any kind of mathematical function, and von Neumann's inspiration in 1946 that such instructions could be stored electronically. The ANN represents an alternative approach in which solutions are arrived at through a training set.

The main feature of an ANN is this ability to determine solutions from examples. For most purposes the network can be regarded as a 'black box' which proposes likely outputs (disease conditions) from inputs (clinical observations). The precise form of the transformation is governed by a network of internal connections between inputs and outputs, modelled on the biological neuron, with the strengths of the synaptic pathways determined through exposure to a training set. Learning can be a computationally intensive undertaking, but once established, new data can be processed very rapidly. For many applications this learning process circumvents the need to develop a principled model of the decision making process, which can often prove difficult or impossible to establish, especially in medical diagnosis.

The origins of neural computing lie in the 1940s with the work of McCulloch and Pitts, ${ }^{1}$ showing that networks of model neurons are, in principle, capable of emulating any general purpose computing machine. The 1960 s saw a great deal of research activity in neural networks, much of it suffering from a lack of rigour, and momentum in the field diminished as some fairly elementary but intractable problems emerged. The resurgence began in the early 1980s with Hopfield's ${ }^{2}$ discovery of new learning algorithms and this, coupled with the failure of other forms of artificial intelligence to live up to expectations, led to an explosion of interest in neural computing. Commercially, ANNs can be found now in consumer electronics and domestic appliances, for applications varying from autoexposure on video cameras to cooking time control for microwave ovens. The main disadvantages of ANNs stem from the need to provide a rich set of comprehensive, nonconflicting examples for training and this may have hindered their general medical application.

The application of ANNs to medical image interpretation has, however, received a great deal of attention in mammography. The goal here is rapid, automatic screening for characteristic patterns of malignant lesions. While a high proportion of the early ophthalmic interest in ANNs has been in visual field assessment, ${ }^{34}$ more recently, extracted structural image features have been added to clinical observations to instantiate discrete diseases, such as glaucoma ${ }^{5-7}$ and the classification of microaneurysms. ${ }^{8}$ Screening aspects are also attracting a small, but increasing amount of attention, for this can be an expensive and time consuming manual process, where the probability of suspicious findings are small but the consequences of delay are important for clinical care. While the primary rationale may be workload driven, it is well known that diagnostic performance degrades rapidly when appraising images. Only with automation, not just at the data collection, but at the detection and classification stages can future, large scale screening programmes survive.

The paper by Gardner and colleagues in this issue of the BFO (p 940) starts with a basic fundus image and, by a selection of straightforward image processing strategies, forms inputs to the ANN so that rules of association which identify vessels, exudates, and haemorrhages can be formulated in the connection weights. They acknowledge that many of the image processing steps have been evaluated previously, but the significance of the work is in assessing these in an automatic, learning framework which is driven by the intrinsic image structure rather than features derived from the images. While the quoted sensitivity and specificity for detection of diabetic retinopathy are each around $85 \%$, one of the most valuable features of the ANN technology is that these operating limits can 
easily be adjusted, improving the true positive detection rate at the expense of more false positive indications.

Of course, we must not get too carried away by this apparent ability to automatically classify by painless learning processes, for while the computing specialist has always seen the necessity to express decision rules in terms of mathematics or logic, the statistician also has an armoury of classification tools able to produce results often as convincing as those of the ANN. Indeed, there is much to commend using the techniques of multivariate distributions and principal component analysis as a silver standard, after the gold standard of the expert, by which to evaluate the new methods. Analytically, the ANN only drives a decision surface through the data, and statisticians claim to have been doing just that for a very long time.

Although it will also be a long time before artificial neural networks approach the complexity or performance of their human counterparts, and in this respect the quoted receiver operator coefficient (ROC) curves often show there is room for improvement, it is clear that the processes of decision making we use on a day to day basis embarrassingly defy an exclusively algorithmic synthesis. The fact that biological systems achieve such impressive feats of information processing using a basic, simplistic, connec- tionist approach will remain a constant source of inspiration. While it would be unwise to speculate on technical developments in this field, there can be little doubt that the future will be an exciting one.

P UNDRILI

Department of Bio-Medical Physics and Bio-Engineering, University of Aberdeen Medical School,

Foresterhill, Aberdeen AB25 2ZD

1 McCulloch WS, Pitts W. A logical calculus of the ideas immanent in nervous activity. Bull Math Biophys 1943;5:115-33.

2 Hopfield JJ. Neural networks and physical systems with emergent collective computational abilities. Proc Natl Acad Sci USA 1984;79:2554-8.

3 Mutlukan E, Keating D. Visual field interpretation with a personal computer based neural network. Eye 1994;8:321-3.

4 Spenceley SE, Henson DB, Bull DR. Visual field analysis using artificial neural networks. Ophthal Physiol Opt 1994;14:239-48.

5 Brigatti L, Hoffman D, Caprioli J. Neural networks to identify glaucoma with structural and functional measurements. Am f Ophthalmol 1996;121: 511-21.

6 Goldbaum MH, Sample PA, White H, Colt B, Raphaelian P, Fechtner RD, et al. Interpretation of automated perimetry for glaucoma by neura network. Invest Ophthalmol Vis Sci 1994;35:3362-73.

7 Madsen EM, Yolton RL. Demonstration of a neural network expert system for recognition of glaucomatous visual field changes. [Review] Mil Med 1994;159:553-7.

8 Frame A, Cree MJ, Olson J, Undrill PE. Computer based classification of retinal microaneurysms. Proceedings of International Conference on Neural Networks and Expert Systems in Health Care, Plymouth, 1996.

\section{Lymphocyte subsets and ocular inflammation: future prospects for immune deviation therapy?}

In the early 1960 s the cells of the immune system were simply divided into the $\mathrm{B}$ cells and the $\mathrm{T}$ cells. $\mathrm{B}$ cells were responsible for antibody production whereas $T$ cells produced a wide variety of soluble factors designated as lymphokines. Subdivision of T cells became possible with the development of the technique to produce monoclonal antibodies against a wide variety of proteins including cell surface components. This led to a classification of $\mathrm{T}$ cells based on the presence of the so called markers CD4 or CD8. Analysis of these two types of $T$ cells resulted in a functional separation of $\mathrm{T}$ cell populations into $\mathrm{CD} 4$ positive $\mathrm{T}$ cells providing a helper function for the differentiation of antibody producing B cells and CD8 positive T cells which were shown to kill target cells and thus were named cytotoxic $T$ cells. Recently it has become clear, however, that such a strict functional division is not valid any more and that certain properties of CD4 and CD8 T cells show an overlap. CD4 cells interact with antigen presenting cells via MHC class II (HLA-DR) molecules, whereas CD8 cells recognise antigen in the context of MHC class I (HLA-A, B, or C).

It soon appeared that the membrane markers were not sufficient to characterise the various functions of $T$ cells, and a further breakthrough came from the functional and molecular characterisation of the many lymphokines produced by these cells. In the late 1980s Mosman and Coffman ${ }^{2}$ presented a division of the $\mathrm{T}$ helper cells into Th1 and Th2 populations, depending upon their lymphokine (cytokine) production profile. The balance between a Th1 or a Th2 response is of great importance in the outcome of the immune defence mechanisms employed to combat infectious disease, and may also influence the occurrence of allergic (conjunctivitis) or autoimmune disease. ${ }^{3}$ This has been nicely illustrated in a mouse model of leishmaniasis whereby mouse strains that react to this infection with a Th2 type response succumb, whereas animals showing a Th1 reactivity survive.
Deviation of the Th2 response in these mouse strains to a Th1 response leads to protection against the Leishmania parasite.

A large body of evidence now shows that a Th1 response is primarily responsible for a delayed type hypersensitivity response and is characterised by a high production of interferon $\gamma$ and IL-2 and a low or absent release of IL-4 (see Table 1). The Th2 cells, on the other hand, produce large amounts of IL-4 and IL-5 and play a key role in humoral immunity including the IgE response. The cytokines produced by the Th2 cells have a deactivating effect on macrophages and thus counteract the tissue destruction which is characteristic of the Th1 response. An exaggerated Th2 response is associated with allergic disease and immunoglobulin mediated autoimmunity, whereas a Th1 response is held responsible for contact hypersensitivity and certain autoimmune diseases such as rheumatoid arthritis. It has been speculated that several organ specific autoimmune diseases are caused by interferon $\gamma$ producing Th1 cells. These findings have led to new approaches for the immunotherapy of inflammatory autoimmune disease which are based on a deviation of the harmful Th1 response towards an immunosuppressive Th2 response.

Our knowledge concerning the factors that regulate the balance between $\mathrm{Th} 1 / \mathrm{Th} 2$ is rapidly expanding. Th2 cell proliferation is inhibited by interferon $\gamma$ and Th1 cell proliferation is suppressed by IL-4. Other cytokines that play a regulatory role are IL-10 and IL-12 which are both produced by antigen presenting cells. IL-10 supports the Th2 response and IL-12 the Th1 response. The microenvironment may dictate whether an antigen presenting cell produces IL-10 or IL-12 thus leading to either a Th1 or a Th2 response. Administration of antigens via the mucosal immune system is thought to induce a Th2 response and so called oral tolerisation strategies are currently being explored to treat a number of putative autoimmune 